\section{Prevalência de desordem coordenativa desenvolvimental em crianças com 7 a 10 anos de idade}

\section{Prevalence of developmental coordination disorder in children aged 7 to 10 years}

Viviane Aparecida Pereira dos Santos

José Luiz Lopes Vieira²

Resumo - Os movimentos das crianças, inicialmente, são caracterizados por ações inconsistentes e imprecisas, mas com a prática e a experiência, os padrões motores tornam-se mais refinados e apresentam melhor coordenação e controle. No entanto, não é raro observar crianças que manifestam certas dificuldades de movimento, podendo interferir nas relações sociais, emocionais, afetivas e escolares das crianças. O estudo investigou a prevalência de Desordem Coordenativa Desenvolvimental (DCD) em escolares com idade entre 7 e 10 anos e, em que tarefas motoras as crianças com provável DCD e risco de DCD apresentam maior dificuldade motora. Foram avaliadas 581 crianças, matriculadas regularmente do $2^{\circ}$ ao $5^{\circ}$ ano do ensino fundamental de escolas públicas de Maringá-Pr, por meio da bateria II e III do teste Movement Assessment Battery for Children (MABC). Os resultados indicaram que $78,1 \%$ das crianças apresentam Desenvolvimento Típico (DT), 10,5\% de crianças com risco de Desordem Coordenativa Desenvolvimental (Risco de DCD) e 11,4\% de crianças diagnosticadas com potencial Desordem Coordenativa Desenvolvimental (Potencial DCD). Portanto, os resultados demonstraram alta prevalência da população para Risco de DCD e potencial DCD, o que prediz níveis desfavoráveis na aquisição e aperfeiçoamento das habilidades fundamentais, podendo, assim, prejudicar a continuidade do processo de desenvolvimento motor das crianças. A maior dificuldade motora encontrada pelas crianças que apresentaram DT foi nas habilidades manuais, enquanto que para as crianças identificadas com provável DCD e risco de DCD foi nas habilidades com bola.

Palavras-chave: Crianças; Desenvolvimento motor; Dificuldade motora; Habilidades motoras.

Abstract - Children's movements are initially characterized by inconsistent and imprecise actions. However, with practice and experience, the motor patterns become more refined and they present better coordination and control. However, it is not rare to observe children that manifest certain movement difficulties that might interfere in the children's emotional, affectionate, school and social relationships. We investigated the prevalence of Developmental Coordination Disorder (DCD) in schoolchildren aged 7 to 10 years and which motor tasks the children with probable DCD and risk of DCD present larger motor difficulty. The evaluation included 581 children registered regularly from the 2 nd to the 5 th grade of Elementary School of public schools of Maringá-PR was conducted through the battery II and III of the Movement Assessment Battery for Children (MABC) test. The results indicated that 78,1\% of the children present Typical Development (DT), 10,5\% presented risk of Developmental Coordination Disorder (Risk of DCD) and 11,4\% were diagnosed with potential Developmental Coordination Disorder (Potential DCD). Therefore, the results showed high prevalence of population for Risk of DCD and Potential DCD, predicting unfavorable levels in the acquisition and improvement of fundamental skills, which may harm the continuity of the process of the children's motor development. Manual skills were the major motor difficulty found in children with DT while ball skills were the major motor difficulty in children with probable DCD and risk of DCD.

Key words: Children; Motor abilities; Motor development; Motor difficulties.
1 Fundação Faculdade de Filosofia Ciências e Letras de Mandaguari. Departamento de Educação Física. Mandaguari, PR. Brasil.

2 Universidade Estadual de Maringá. Programa Associado de Pós-Graduação em Educação Física da Universidade Estadual de Maringá e Universidade Estadual de Londrina. Maringá, PR. Brasil.

Recebido em 30/04/12 Revisado em 10/07/12 Aprovado em 20/08/12

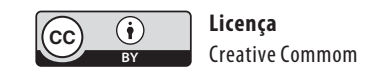




\section{INTRODUÇÃO}

O desenvolvimento da criança encontra nas manifestações motoras sua principal forma de expressão. O movimento é o meio mais básico pelo qual a criança interage de forma dinâmica com o ambiente físico e social. Movimento e criança se confundem, seja executando habilidades motoras finas ou amplas, seja manipulando instrumentos ou se deslocando, movimentar-se é uma constante no universo da criança ${ }^{1}$. O ato de explorar o movimento no brincar é algo que se pode perceber no cotidiano do mundo infantil. As crianças improvisam e experimentam brincando, o que gera diversidade de movimento no seu repertório motor ${ }^{2}$.

Assim, o desenvolvimento motor na infância caracteriza-se pela aquisição de um amplo repertório de habilidades motoras, que possibilita à criança o domínio do seu corpo em diferentes posturas (estáticas e dinâmicas), locomover-se pelo ambiente de variadas formas (andar, correr, saltar e outros) e manipular objetos e instrumentos diversos (receber uma bola, arremessar, chutar e outros) ${ }^{3}$. Essas habilidades básicas direcionam o aprendiz para a realização das atividades do dia-a-dia e constituem o meio de aperfeiçoamento para habilidades mais complexas. Enquanto os movimentos dos aprendizes são, inicialmente, caracterizados por ações inconsistentes e imprecisas, com a prática e a experiência, os padrões motores tornam-se mais refinados e apresentam melhor coordenação e controle em comportamentos habilidosos.

Entretanto, não é raro observar crianças que manifestam certas dificuldades quando interagem com o meio em que vivem. Assim, quando essas dificuldades não são identificadas, podem interferir nas relações sociais, emocionais, afetivas e escolares das crianças. No contexto escolar, crianças com dificuldades motoras são excluídas por seus colegas em brincadeiras e jogos, por não apresentarem competências suficientes nos movimentos motores. Deste modo, crianças com atrasos motores tendem a evitar qualquer experiência que demande uma boa coordenação motora, levando-as a não participação de atividades físicas, tanto no lazer como nas atividades escolares ${ }^{4}$. Crianças com estas características de comportamento motor destacam-se das demais de mesma faixa etária, podendo apresentar o que tem sido denominado de Desordem Coordenativa Desenvolvimental $\mathrm{DCD}^{5}$. Essas crianças com insuficiência de coordenação em experiências motoras, jogos, brincadeiras do cotidiano e, até mesmo, em atividades desenvolvidas nas aulas de Educação Física na escola, apresentam dificuldades quando solicitadas a executar tarefas motoras, aparentemente simples, tais como correr e saltar consecutivamente, saltar e arremessar simultaneamente, entre outras, quando comparadas a crianças da mesma faixa etária ${ }^{6}$. Essas crianças apresentam atraso motor nos padrões motores básicos, geralmente, sendo consideradas como descoordenadas tanto em habilidades motoras grossas quanto nas habilidades motoras finas ${ }^{7}$. Esse atraso motor a curto e longo prazo interfere na qualidade da vida de crianças com DCD, sendo essencial a identificação precoce e seu encami- 
nhamento para programas de intervenção que minimizem os problemas decorrentes da desordem ${ }^{8,9}$.

A Incidência de DCD tem sido relatada pela literatura, com estimativa de 2 a 19\% pelos países, causando uma preocupação mundial quanto à qualidade dos serviços oferecidos a essas crianças ${ }^{10}$. Estudos na Alemanha e na Holanda evidenciaram 7,7\% de DCD, entre crianças de quatro a 13 anos $^{11}$, na Suíça $7,3 \%{ }^{12}$, na Inglaterra $1,7 \%$ de crianças com provável DCD e $4,9 \%$ em situação de risco para $\mathrm{DCD}^{13}$, para crianças com 7 anos de idade. Ainda, um estudo cross-cultural conduzido no Canadá constatou $8 \%$ enquanto que, na Grécia, a estimativa foi de $19 \%{ }^{14}$. Em estudos conduzidos no Brasil, as estimativas variam em torno de $4,4 \%$ a $19,9 \%$. Por exemplo, um estudo conduzido em Manaus reportou na zona rural 4,4\% e 11,8\% na zona urbana, para crianças com 7 e 8 anos com provável DCD². Já, outro estudo conduzido na região sul do país, com 1587 crianças, com idade entre quatro e 12 anos, demonstrou 19,9\% de $\mathrm{DCD}^{10}$.

Desta forma, o objetivo desse estudo foi investigar a prevalência de potencial DCD em crianças com idade entre 7 e 10 anos, matriculadas do $2^{\circ}$ ao $5^{\circ}$ ano do ensino fundamental de escolas públicas da cidade de Maringá-Pr e, ainda, investigar em que tarefas motoras as crianças com provável DCD e risco de DCD apresentam maior dificuldade motora.

\section{PROCEDIMENTOS METODOLÓGICOS}

A amostra foi constituída por 581 crianças, com idade entre 7 e 10 anos, sendo 159 crianças com 7 anos de idade (77 do gênero feminino e 82 do gênero masculino), 153 crianças de 8 anos ( 83 do gênero feminino e 70 do gênero masculino ), 173 crianças de 9 anos ( 86 do gênero feminino e 87 do gênero masculino) e 96 crianças de 10 anos de idade (38 do gênero feminino e 58 do gênero masculino) matriculadas no ensino fundamental de escolas públicas do município de Maringá do estado do Paraná. Entre as crianças selecionadas, 247 (42,5\%) foram de instituições educacionais da região nordeste, 142 $(24,4 \%)$ da região sudeste, $129(22,2 \%)$ da região noroeste e $63(10,9 \%)$ da região sudoeste do município de Maringá. É importante ressaltar que se optou por este design amostral devido à característica da pesquisa (prevalência). Foram critérios para a seleção da amostra, a idade entre 7 e 10 anos e estar matriculado do $2^{\circ}$ ao $5^{\circ}$ ano do ensino fundamental das instituições públicas selecionadas em cada região da cidade de Maringá. Crianças com diagnóstico prévio de neuropatologias e dificuldade motoras foram excluídas do presente estudo. A seleção das escolas do ensino fundamental foi aleatória, por meio de sorteio, assim como a seleção das crianças, com autorização do Núcleo Regional de Maringá, da Secretaria Municipal de Educação, e dos pais ou responsáveis legais por meio do Termo de Consentimento Livre e Esclarecido. Esse estudo foi submetido à aprovação do Comitê de Ética em Pesquisa envolvendo Seres Humanos da Universidade Estadual de Maringá (Protocolo 135/2008).

Como instrumento de medida para verificar o desempenho motor das crianças foi utilizado o Teste MABC - Movement Assessment Battery 
for Children ${ }^{15}$. Foram utilizadas duas baterias do Teste: a Bateria II, para crianças de 7 e 8 anos de idade e a Bateria III para crianças com 9 e 10 anos de idade.

O Teste MABC é composto de três subtestes motores, envolvendo habilidades manuais, de equilíbrio estático e dinâmico e com bola. Cada subteste é composto de oito tarefas motoras. Os valores brutos obtidos em cada uma das tarefas motoras são somados e convertidos em escores de zero (0) a cinco (5) para cada subteste (escores mais elevados representam maiores dificuldades motoras). A soma dos escores de cada domínio fornece o valor do escore total de prejuízo motor, que é convertido em percentil. Pontos de corte reconhecidos na literatura ${ }^{16}$ foram adotados: escores $\leq 5 \%$ representam um desempenho motor atípico indicativo de Desordem Coordenativa Desenvolvimental (DCD); percentil de 6\% a 15\% considerado desempenho motor suspeito (risco de DCD), e percentil $>16 \%$ considerado como Desempenho Típico (DT).

A avaliação foi conduzida nas escolas por 4 profissionais de educação física treinados, com experiência mínima de 3 anos em avaliação e diagnóstico percepto-motor. Para a análise de confiabilidade dos avaliadores, escolheram-se, aleatoriamente, 24 crianças da amostra, distribuídas entre idades de 7 a 10 anos, sendo 6 para cada idade. Os 4 avaliadores treinados para a coleta de dados, cada um deles, aplicou o teste nessas crianças e o resultado foi anotado. E para verificar a existência ou não de diferença entre os grupos, aplicou-se o teste de Friedman, adotando significância de 5\%. A confiabilidade interobservador foi elevada $(0,98)$.

A avaliação de cada criança levou, em média, 25 minutos. As crianças, primeiramente, receberam instrução verbal e demonstração das tarefas motoras da bateria. Quando não compreendida a tarefa, nova explicação foi oportunizada.

$\mathrm{Na}$ análise estatística, verificou-se a normalidade da distribuição dos dados utilizando o teste de Shapiro-Wilk. Foi utilizada estatística descritiva para verificar a prevalência de potencial DCD, Risco de DCD e DT, por meio de frequência relativa e absoluta. Para a comparação entre idades, foi utilizado o Teste de Homogeneidade de Levene, indicando homogeneidade dos grupos, optou-se pelo Teste Anova One-Way com Post Hoc de Bonferroni. Quando o Teste de Homogeneidade de Levene não assumia a homogeneidade dos grupos, utilizou-se o Teste Kruskal-Wallis com Post Hoc U de Mann-Whitney. Para comparação entre as habilidades manuais, com bola e de equilíbrio, foi utilizado o Teste ANOVA de Medidas Repetidas com Esfericidade de Maucly's, quando a esfericidade era assumida, e quando a esfericidade não era assumida optou-se pela correção de Greenhouse-Geisser com Post Hoc de Bonferroni. A significância adotada foi de 5\% e o software foi o SPSS 13.0.

\section{RESULTADOS}

No total da amostra, ou seja, para todas as crianças, foi identificado por meio da frequência relativa (\%) que 78,1\% das crianças demonstraram DT, 11,4\% 
das crianças apresentaram provável DCD e 10,5\% risco de DCD. A Tabela 1 demonstra, ainda, que as crianças com 7 e 10 anos foram as que apresentaram maior prevalência para possível DCD, enquanto que, para risco de DCD, a maior prevalência ocorreu para as crianças com 8 e 9 anos de idade.

Tabela 1. Frequência absoluta (F) e frequência relativa (\%) da prevalência e risco de DCD e desenvolvimento típico.

\begin{tabular}{lccccccccccc}
\hline & \multicolumn{10}{c}{ Idade } \\
\cline { 2 - 14 } Prevalência & \multicolumn{1}{c}{7 anos } & \multicolumn{2}{c}{8 anos } & \multicolumn{2}{c}{9 anos } & \multicolumn{2}{c}{10 anos } & \multicolumn{2}{c}{ Total } \\
\cline { 2 - 14 } & F & $\%$ & $F$ & $\%$ & $F$ & $\%$ & $F$ & $\%$ & $F$ & $\%$ \\
\hline DT & 119 & 074,8 & 124 & 081,1 & 134 & 077,4 & 77 & 080,2 & 454 & 078,1 \\
Risco DCD & 014 & 008,8 & 021 & 013,7 & 020 & 011,6 & 06 & 006,3 & 061 & 010,5 \\
DCD & 026 & 016,4 & 008 & 005,2 & 019 & 011,0 & 13 & 013,5 & 066 & 011,4 \\
Total & 159 & 100,0 & 153 & 100,0 & 173 & 100,0 & 96 & 100,0 & 581 & 100,0 \\
\hline
\end{tabular}

DT (Desenvolvimento Típico); Risco DCD (Risco de Desordem Coordenativa Desenvolvimental); DCD (Potencial Desordem Coordenativa Desenvolvimental).

$\mathrm{Na}$ Tabela 2, em função da não normalidade dos dados, os resultados serão apresentados em Mediana (MD) e $1^{\circ}$ e $3^{\circ} \mathrm{Quartis}\left(1^{\circ} \mathrm{Q}\right.$ e $\left.3^{\circ} \mathrm{Q}\right)$, seguidos da significância encontrada ( $\mathrm{p}=$ valor) evidenciada pela variável DT. Esta diferença ocorreu entre as crianças de 8 e 9 anos ( $p=0,0001)$ e 8 e 10 anos $(\mathrm{p}=0,008)$ enquanto que entre as crianças de 7 e 8 anos a significância foi de $\mathrm{p}=0,127$, indicando que as crianças de 8 anos de idade apresentaram desempenho motor superior quando comparadas às crianças das demais idades. E para as crianças com potencial DCD verifica-se que não houve diferença significativa, porém, os grupos etários de 7 e 10 anos constataram, por meio da mediana (16,5 e 18,0 pontos, respectivamente), índices maiores de dificuldade motora que as crianças de 8 e 9 anos. O mesmo ocorreu para as crianças que apresentaram risco de DCD.

Tabela 2. Comparação para o desenvolvimento típico (DT), risco de desordem coordenativa desenvolvimental (Risco de DCD) e potencial desordem coordenativa desenvolvimental (DCD).

\begin{tabular}{lccccc}
\hline & \multicolumn{5}{c}{ Idade } \\
\cline { 2 - 5 } Prevalência & 7 anos & 8 anos & 9 anos & 10 anos & P-valor \\
\cline { 2 - 5 } & MD $\left(1^{\circ} \mathrm{Q}-3^{\circ} \mathrm{Q}\right)$ & $\mathrm{MD}\left(1^{\circ} \mathrm{Q}-3^{\circ} \mathrm{Q}\right)$ & $\mathrm{MD}\left(1^{\circ} \mathrm{Q}-3^{\circ} \mathrm{Q}\right)$ & $\mathrm{MD}\left(1^{\circ} \mathrm{Q}-3^{\circ} \mathrm{Q}\right)$ & \\
\hline DT & $5,0(2,5-7,0)$ & $3,5(1,5-6,0)$ & $5,5(3,0-7,0)$ & $5,0(3,5-6,7)$ & $0,0001^{*}$ \\
Risco DCD & $12,2(10,8-12,5)$ & $11,5(10,7-12,0)$ & $11,5(11,0-12,5)$ & $12,2(10,8-12,5)$ & $0,6470^{*}$ \\
\hline DCD & $16,5(14,3-18,1)$ & $15,7(14,7-18,0)$ & $16,0(14,5-18,0)$ & $18,0(15,2-21,0)$ & $0,4210^{*}$ \\
\hline
\end{tabular}

${ }^{*} p<0,001$ MD (Mediana); $\mathrm{Q}$ (Quartil).

$\mathrm{Na}$ Tabela 3, encontram-se as comparações realizadas entre as crianças de 7 a 10 anos de idade para DCD, Risco de DCD e DT referentes às habilidades manuais (HM), habilidades com bola (HB) e habilidades de equilíbrio (HE), visando constatar para cada uma dessas habilidades qual ou quais grupos etários demonstram maior dificuldade motora. 
Tabela 3. Comparação para desenvolvimento típico, risco de DCD e potencial DCD referentes às habilidades manuais, com bola e de equilíbrio.

\begin{tabular}{|c|c|c|c|c|c|}
\hline & \multicolumn{5}{|c|}{ Desenvolvimento típico } \\
\hline & 7 anos & 8 anos & 9 anos & 10 anos & \multirow{2}{*}{$P$-valor } \\
\hline & $\mathrm{MD}\left(1^{\circ} \mathrm{Q}-3^{\circ} \mathrm{Q}\right)$ & $\mathrm{MD}\left(1^{\circ} \mathrm{Q}-3^{\circ} \mathrm{Q}\right)$ & $\mathrm{MD}\left(1^{\circ} \mathrm{Q}-3^{\circ} \mathrm{Q}\right)$ & $\mathrm{MD}\left(1^{\circ} \mathrm{Q}-3^{\circ} \mathrm{Q}\right)$ & \\
\hline $\mathrm{HM}$ & $0,83(0,00-1,33)$ & $0,58(0,04-1,33)$ & $0,50(0,17-1,21)$ & $0,67(0,17-1,25)$ & $0,9690^{*}$ \\
\hline $\mathrm{HB}$ & $0,50(0,00-1,50)$ & $0,50(0,00-1,00)$ & $0,00(0,00-1,00)$ & $0,00(0,00-0,75)$ & $0,002 * 0$ \\
\hline \multirow[t]{2}{*}{ HE } & $0,00(0,00-0,17)$ & $0,00(0,00-0,17)$ & $0,50(0,17-1,00)$ & $0,33(0,17-0,91)$ & $0,0001^{*}$ \\
\hline & \multicolumn{5}{|c|}{ Risco DCD } \\
\hline $\mathrm{HM}$ & $1,50(1,17-2,00)$ & $2,00(1,67-2,33)$ & $1,91(1,04-2,45)$ & $1,75(0,54-2,79)$ & $0,3870^{*}$ \\
\hline $\mathrm{HB}$ & $2,00(1,00-3,50)$ & $1,50(0,87-2,37)$ & $1,25(0,12-2,50)$ & $1,75(1,37-2,50)$ & $0,035 * 0$ \\
\hline \multirow[t]{2}{*}{$\mathrm{HE}$} & $0,67(0,00-1,00)$ & $0,33(0,08-1,25)$ & $1,00(0,50-1,62)$ & $0,75(0,00-2,08)$ & $0,0800^{*}$ \\
\hline & \multicolumn{5}{|c|}{ DCD } \\
\hline $\mathrm{HM}$ & $2,50(1,33-3,17)$ & $2,58(1,75-2,95)$ & $2,67(1,83-3,67)$ & $1,83(1,17-3,08)$ & $0,7470^{*}$ \\
\hline $\mathrm{HB}$ & $2,75(2,25-3,50)$ & $3,12(2,25-3,50)$ & $2,00(0,50-3,00)$ & $4,00(2,50-4,50)$ & $0,003 * 0$ \\
\hline $\mathrm{HE}$ & $1,41(0,67-1,83)$ & $1,08(0,21-1,45)$ & $2,00(1,00-2,67)$ & $1,50(1,25-1,91)$ & $0,0530^{*}$ \\
\hline
\end{tabular}

Risco DCD (Risco de Desordem Coordenativa Desenvolvimental); DCD (Potencial Desordem Coordenativa Desenvolvimental); MD (Mediana); Q (Quartil).

Para as crianças que apresentaram DT, foi constatada diferença significativa para as $\mathrm{HB}$ e HE. Para a $\mathrm{HB}$, observou-se pior desempenho para as crianças com 7 anos. Já para a HE o pior desempenho foi evidenciado para as crianças com 9 e 10 anos. Para as crianças que apresentaram Risco de DCD, foi averiguado diferença significativa apenas para a HB, evidenciando pior desempenho para as crianças de 7 e 8 anos. E, por fim, para as crianças que demonstraram provável DCD, observou-se diferença para a $\mathrm{HB}$, constatando pior desempenho para as crianças com 10 anos.

$\mathrm{Na}$ Tabela 4, serão apresentadas as comparações entre as HM, HB e HE, com o objetivo de identificar em qual, ou em quais habilidades motoras as crianças de 7 a 10 anos de idade com potencial DCD, Risco de DCD e DT apresentaram maior dificuldade motora.

Observa-se, na Tabela 4, para as crianças com 7 anos de idade, dife-

Tabela 4. Comparação entre as Habilidades Manuais, com Bola e de Equilíbrio referente aos escolares de 7 a 10 anos de idade.

\begin{tabular}{|c|c|c|c|c|c|}
\hline \multirow{3}{*}{ Idades } & \multirow{3}{*}{ Prevalência } & \multicolumn{4}{|c|}{ Habilidades } \\
\hline & & Habilidades Manuais & Habilidades com Bola & Habilidades de Equilíbrio & $P_{\text {-valor }}$ \\
\hline & & $\mathrm{MD}\left(1^{\circ} \mathrm{Q}-3^{\circ} \mathrm{Q}\right)$ & $\mathrm{MD}\left(1^{\circ} \mathrm{Q}-3^{\circ} \mathrm{Q}\right)$ & $\mathrm{MD}\left(1^{\circ} \mathrm{Q}-3^{\circ} \mathrm{Q}\right)$ & $p-v d i o f$ \\
\hline \multirow{3}{*}{7 anos } & DT & $0,83(0,00-1,33)$ & $0,50(0,00-1,50)$ & $0,00(0,00-0,17)$ & $0,0001^{*}$ \\
\hline & Risco DCD & $1,50(1,17-2,00)$ & $2,00(1,00-3,50)$ & $0,67(0,00-1,00)$ & $0,003 * 0$ \\
\hline & DCD & $2,50(1,33-3,17)$ & $2,75(2,25-3,50)$ & $1,41(0,67-1,83)$ & $0,003 * 0$ \\
\hline \multirow{3}{*}{8 anos } & DT & $0,58(0,04-1,33)$ & $0,50(0,00-1,00)$ & $0,00(0,00-0,17)$ & $0,0001^{*}$ \\
\hline & Risco DCD & $2,00(1,67-2,33)$ & $1,50(0,87-2,37)$ & $0,33(0,08-1,25)$ & $0,0001^{*}$ \\
\hline & DCD & $2,58(1,75-2,95)$ & $3,12(2,25-3,50)$ & $1,08(0,21-1,45)$ & $0,0530^{*}$ \\
\hline \multirow{3}{*}{9 anos } & DT & $0,50(0,17-1,21)$ & $0,00(0,00-1,00)$ & $0,50(0,17-1,00)$ & $0,003 * 0$ \\
\hline & Risco DCD & $1,91(1,04-2,45)$ & $1,25(0,12-2,50)$ & $1,00(0,50-1,62)$ & $0,0500^{*}$ \\
\hline & DCD & $2,67(1,83-3,67)$ & $2,00(0,50-3,00)$ & $2,00(1,00-2,67)$ & $0,0530^{*}$ \\
\hline \multirow{3}{*}{$\begin{array}{l}10 \\
\text { anos }\end{array}$} & DT & $0,67(0,17-1,25)$ & $0,00(0,00-0,75)$ & $0,33(0,17-0,91)$ & $0,018^{*} 0$ \\
\hline & Risco DCD & $1,75(0,54-2,79)$ & $1,75(1,37-2,50)$ & $0,75(0,00-2,08)$ & $0,3110^{*}$ \\
\hline & DCD & $1,83(1,17-3,08)$ & $4,00(2,50-4,50)$ & $1,50(1,25-1,91)$ & $0,002 * 0$ \\
\hline
\end{tabular}

DT (Desenvolvimento Típico); Risco DCD (Risco de Desordem Coordenativa Desenvolvimental); DCD (Potencial Desordem Coordenativa Desenvolvimental). 
renças significativas para todas as crianças. Para as crianças que apresentaram DT, constatou-se maior dificuldade motora para as $\mathrm{HM} \mathrm{e} \mathrm{HB,} \mathrm{com}$ piores resultados para as HM. Para as crianças com provável DCD e risco de $\mathrm{DCD}$, maiores dificuldades motoras foram encontradas para as $\mathrm{HM}$ e $\mathrm{HB}$, evidenciando pior desempenho para a HB. Já para as crianças com 8 anos de idade, ocorreram diferenças estatísticas para as crianças com DT e risco de DCD, constatando-se pior desempenho para as HM e HB, prevalecendo resultados inferiores para a HM. Para as crianças com 9 anos, evidenciaram-se diferenças significativas para as crianças com DT, demonstrando pior desempenho para a HM seguida da HE. E, por fim, para as crianças com 10 anos de idade, observaram-se diferenças significativas para DT e provável DCD. Para as crianças com DT, o pior desempenho foi identificado para a HM e para as crianças com provável DCD, o pior desempenho foi constatado para a HB. Os resultados encontrados demonstram que as crianças entre 7 e 10 anos de idade com DT apresentaram maior dificuldade motora para as HM, exceto as de 9 anos que também apresentaram dificuldade para as HE, enquanto que as crianças com provável $\mathrm{DCD}$ e risco de DCD evidenciaram pior desempenho para as $\mathrm{HB}$, exceto as de 8 anos que apresentaram risco de DCD. Estas evidenciaram pior desempenho para as HM.

\section{DISCUSSÃO}

A prevalência estimada na população infantil entre 5 e 11 anos de idade para potencial DCD é de $6 \% 5,17$. No entanto, os estudos realizados em diversos países apontam para um índice de prevalência variando entre $2,7 \%{ }^{18}$ a $15,6 \%{ }^{19}$.

Ainda, outros estudos ${ }^{4,20-22}$ apontam uma estimativa de 5 a $8 \%$ de dificuldades motoras, variando estes resultados pelos países. Mas, dependendo dos critérios utilizados, pode-se obter em torno de 5 a 15\% de crianças de uma determinada população com provável $\mathrm{DCD}^{23}$. Outro estudo conduzido na Grécia encontrou uma estimativa de $19 \%{ }^{14}$, considerado superior aos resultados encontrados em outras pesquisas.

Em países como a Inglaterra, por exemplo, 10\% das crianças têm sido reportadas com dificuldades motoras. Na população Norte Americana, 5 a $10 \%$ das crianças em idade escolar são estimadas com provável $\mathrm{DCD}^{24}$. $\mathrm{Na}$ Europa, a estimativa é de 5 a $8 \%$ da população escolar que possuem o desenvolvimento da competência motora abaixo do progresso normal nas atividades escolares gerais e particularmente, na Educação Física ${ }^{17}$. Mas, hoje, a prevalência tem apontado para $5 \%$ da população infantil, com um adicional na ordem de $10 \%$ para uma categoria de risco (Risco de DCD) ${ }^{17}$.

No Brasil, poucos estudos têm sido realizados para identificar as dificuldades motoras de crianças. Em Manaus, em um estudo ${ }^{2}$ constituído por 240 crianças de 7 e 8 anos de idade, da zona urbana e rural, 11,8\% das crianças da zona urbana foram classificadas com provável DCD e 10,3\% como grupo de risco (Risco de DCD). Na zona rural, 4,4\% das crianças 
foram classificadas com potencial DCD e 11,1\% como grupo de risco (Risco de DCD). Outro estudo conduzido na região sul do país, com 1587 crianças com idade entre quatro e 12 anos, demonstrou $19,9 \%$ de $\mathrm{DCD}^{10}$.

Deste modo, os resultados encontrados nesse estudo não excederam os valores encontrados na literatura brasileira, demonstrando que, da amostra total do estudo, $11,4 \%(\mathrm{n}=66)$ das crianças apresentaram dificuldades motoras indicando potencial DCD, 10,5\% ( $\mathrm{n}=61)$ das crianças Risco de DCD e 78,1\% ( $\mathrm{n}=454)$ não foram identificadas dificuldades motoras, classificando-se com Desenvolvimento Típico. De acordo com a frequência identificada para os grupos etários, verificou-se que, para as crianças de 7 anos, o problema motor (DCD) está mais evidente, com 16,4\% ( $\mathrm{n}=26)$, seguido dos 10 anos, 13,5\% (n=13), 9 anos, $11,0 \%(n=19)$ e 8 anos de idade, $5,2 \%(\mathrm{n}=8)$.

Os resultados encontrados para as comparações entre as idades referentes às habilidades motoras (habilidade manual, com bola e de equilíbrio) demonstraram maiores dificuldades para a habilidade com bola, destacando esta dificuldade para as crianças com 7 e 10 anos. Já, para a análise específica entre as habilidades manuais, com bola e de equilíbrio, referentes às crianças de 7 a 10 anos (Tabela 4), verificou-se, para as crianças que apresentaram DT, maior dificuldade para as tarefas que envolvem as habilidades manuais. Diferentemente, para as crianças que apresentaram provável DCD e risco de DCD, a maior dificuldade motora encontrada foi nas tarefas que envolvem as habilidades com bola. Pesquisadores ${ }^{10,25,26}$ relatam em seus estudos maior dificuldade motora para todas as crianças envolvidas nos estudos, ou seja, para as crianças que apresentaram DT, possível DCD e risco de DCD, para as habilidades manuais. Ainda, quando comparado os gêneros, a maior dificuldade motora está nas habilidades com bola, destacando as meninas como mais prejudicadas ${ }^{10}$.

$\mathrm{O}$ atraso no desenvolvimento das habilidades manuais por crianças com DCD e risco de DCD é referido na literatura ${ }^{27}$. A realização dessas tarefas envolve habilidades perceptivas, nas quais crianças com DCD apresentaram dificuldades ${ }^{27}$, o que pode resultar como consequência em déficits na escolarização ${ }^{15,27}$. Com o passar dos anos, as dificuldades tendem a aumentar, sendo imprescindível a avaliação e intervenção continuada por parte da escola e dos familiares ${ }^{10}$.

\section{CONCLUSÕES}

Portanto, os resultados do estudo demonstraram alta prevalência da população para Risco de DCD e potencial DCD, o que é altamente preocupante, considerando os prejuízos que podem ocorrer nas relações sociais, emocionais, afetivas e escolares das crianças e, ainda, considerando os poucos recursos no país para o atendimento interventivo destas.

Quanto às dificuldades nas destrezas motoras, as crianças com potencial DCD e risco de DCD evidenciaram maior dificuldade para as habilidades com bola e manual, o que enfatiza a necessidade de identificar esta 
desordem antes dos anos escolares a fim de melhorar a habilidade manual e com bola, mantendo a continuidade interventiva durante os anos escolares, para que estas dificuldades não evoluam, ocasionando problemas motores e psicológicos maiores.

É importante ressaltar a necessidade de estudos científicos em diferentes áreas que investiguem a natureza da DCD, e que envolvam outros processos avaliativos a fim de se obter resultados mais argumentativos e conscientizar pais e professores sobre esta desordem para a melhoria do cuidado com estas crianças.

\section{REFERÊNCIAS BIBLIOGRÁFICAS}

1. Manoel E. Criança e desenvolvimento: Algumas notas numa perspective etária. In: Krebs RJ, Ferreira Neto CA, organizadores. Tópicos em desenvolvimento motor na infância e adolescência. Rio de Janeiro: Lecsu; 2007. p. 187-99

2. Souza C, Ferreira L, Catuzzo MT, Corrêa UC. O teste ABC do movimento em crianças de ambientes diferentes. Rev Port Cien Desp 2007;7(1):36-47.

3. Santos S, Dantas L, Oliveira JA. Desenvolvimento motor de crianças, de idosos e de pessoas com transtorno da coordenação. Rev Paul Educ Fís 2004;18:33-44.

4. Wright HC, Sudgen DA. The nature of developmental coordination disorder: inter and intra-group differences. Adapt Phys Activ Q 1996;13:357-71.

5. American Psychiatric Association. Manual Diagnóstico e estatístico de transtornos mentais (DSM-IV-TR). Trad. Cláudia Dornelles. 4 ed. Porto Alegre: Artmed, 1994.

6. Petersen R, Oliveira MA. Desordens motoras na aprendizagem do esporte. In: Gaya A, Marques A, Tani G. Desporto para crianças e jovens: razões e finalidades. Porto Alegre: UFRGS; 2004. p. 21-56.

7. Wright HC. Children with development co-ordination disorder - a review. Eur J Phys Educ 1997;2:5-22.

8. Missiuna C, Moll S, King S, King G, Law M. A trajectory of troubles: parents' impressions of the impact of developmental coordination disorder. Phys Occup Ther Pediatr 2007;27:81-101.

9. Gibbs J, Appleton J, Appleton R. Dyspraxia or developmental coordination disorder? Unravelling the enigma. Arch Dis Child 2007;92:534-9.

10. Valentini NC, Coutinho, MTC, Pansera SM, Santos VAP, Vieira JLL, Ramalho $\mathrm{MH}$ et al. Prevalence of motor deficits and developmental coordination disorders in children from South Brazil. Rev Paul Pediatr 2012;30(3):377-84.

11. Jongmans MJ, Smits-Engelsman BC, Shoemaker MM. Consequences of comorbidity of developmental coordination disorders and learning disabilities for severity and pattern of perceptual-motor dysfunction. J Learn Disabil 2003;36:528-37.

12. Kadesjö B, Gillberg C. Attention deficits and clumsiness in Swedish 7-year-old children. Dev Med Child Neurol1998;40:796-04.

13. Lingam R, Hunt L, Golding J, Jongmans M, Emond A. Prevalence of developmental coordination disorder using the DSM-IV at 7 years of age: a UK population-based study. Pediatrics 2009;123:693-700.

14. Tsiotra GD, Flouris AD, Koutedakis Y, Faught BE, Nevill AM, Lane AM et al. A comparison of developmental coordination disorder prevalence rates in Canadian and Greek children. J Adolesc Health 2006;39:125-7.

15. Henderson SE, Sugden D A. Movement Assessment Battery for Children. London: Psychological Corporation; 1992.

16. Geuze RH, Jongmans MJ, Schoemaker MM, Smiths-Engelsman BCM. Clinical and research diagnostic criteria or developmental coordination disorder: a review and discussion. Hum Movement Sci 2001;20:7-47.

17. Sugden DA, Wright HC. Motor coordination disorders in children. London: Sage 
Publication; 1998.

18. Van Dellen T, Vaessen W, Schoemaker MM. Clumsiness definition and selection of subjects. In: Kalverboer AF, editor. Developmental biopsychology: experimental and observation studies in children at risk. Ann Arbor: University of Michigan Press; 1990. p. 223-40.

19. Wright H, Sugden D, Ng R, Tan J. Identification of children with movement problems in Singapore: usefulness of the movement ABC checklist. Adapt Phys Activ Q 1994;11:150-7.

20. Polatajko HJ, Macnab JJ, Anstett B, Malloy-Miller T, Murphy K, Noh S. A clinical trial of the process-oriented treatment approach for children with developmental co-ordination disorder. Dev Med Child Neurol 1995;37(4):310-9.

21. Dewey D, Wilson BN. Developmental coordination disorder: what is it? Phys Occup Ther Pediatr 2001;20(2-3):5-27.

22. Maldonado-Durán M, Glinka J. Motor Skills Disorder. dec. 2006. Available from:<http://www.emedicine.com/ped/topic2640.htm> [2010 abr 20].

23. Wilson PH. Practitioner Review: Approaches to assessment and treatment of children with DCD: an evaluative review. J Child Psychol Psyc 2005; 46(8): 806-23.

24. Kadesjo B, Gillberg C. Developmental coordination disorder in Swedish 7-year-old children. J Am Acad Child Adolesc Psychiatry 1999;38(7):820-8.

25. Smits-Engelsman BCM, Niemeijer AS, Van Galen GP. Fine motor deficiencies in children diagnosed as DCD based on poor grapho-motor ability. Hum Mov sci 2001;20:161-82.

26. Niemeijer AS, Smits-Engelsman BCM, Schoemaker MM. Neuromotor task training for children with developmental coordination disorder: a controlled trial. Dev Med Child Neurol 2007;49(6):406-11.

27. Feder KP, Majnemer A. Handwriting development, competency, and intervention. Dev Med Child Neurol 2007;49:312-7.

\section{Endereço para correspondência}

Viviane Aparecida Pereira dos Santos Rua Bernardino de Campos, 950 Vila Santo Antônio.

CEP: 87030-160 Maringá, PR. Brasil. E-mail:viviaps@hotmail.com 